\title{
Typologie d'après LiDAR des structures agraires et parcellaires fossilisées sous couvert forestier en Lorraine
}

\section{Typology shown by LiDAR of fossilized agrarian features and field systems under forest cover in Lorraine}

\author{
Murielle Georges-Leroy ${ }^{1}$
}

${ }^{1}$ Conservatrice générale du patrimoine, Inspection des patrimoines - collège de l'archéologie, UMR 6249 Chronoenvironnement, murielle.leroy@culture.gouv.fr

RÉSUMÉ. Fréquemment mises en évidence par les télédétections LiDAR aéroportées, les structures agraires et parcellaires sont conservées en forêt sous forme de micro-relief, sur des surfaces considérables. La variété des formes sous lesquelles elles se présentent n'est pas sans soulever des questions sur leur origine et leur fonction. Avec la multiplication des levés LiDAR depuis les années 2000, la nécessité de mettre en place des référentiels d'interprétation est assez vite apparue. Nous proposons ici de présenter la typologie des vestiges agraires et parcellaires conservés dans des forêts en Lorraine, à partir de l'étude de quatre missions LiDAR ayant porté sur environ $300 \mathrm{~km}^{2}$. Une approche de ces vestiges en trois étapes a été élaborée : 1) identification des formes élémentaires, notamment telles qu'elles apparaissent sur les visualisations LiDAR ; 2) étude de la morphogenèse des vestiges, à partir des observations au sol et de quelques sondages ayant permis de mieux appréhender leur structure et leur taphonomie ; 3) analyse morphologique des organisations parcellaires qui contribue à l'interprétation de certaines de ces structures. Cette analyse morphologique et fonctionnelle est complétée par une approche chronologique.

ABSTRACT. Frequently highlighted by remote sensing airborne laser scanning, the agrarian features and field systems are preserved in the forest, in the form of microrelief, covering large areas. The range of forms in which they are shown raises the question as to their origin and their function. With the increasing number of lidarLiDAR surveys since the 2000's the need to set up a framework for interpretation quickly became apparent. We propose here to present the typology of agrarian and field system remains preserved in the Lorraine forests based on a study consisting of four lidarLiDAR surveys over an area of about $300 \mathrm{~km}^{2}$. A three-step approach to these remains has been developed: 1) identification of elementary forms, particularly those which are revealed by the visualization of lidarLiDAR data; 2) study of the morphogenesis of the remains, based on observations on the ground and some excavations enabling us to gain more insight into their features and their taphonomy; 3) morphological analysis of the field systems which contributes to the interpretation of some of these morphological features. This analysis is completed by a chronological approach.

MOTS-CLÉS. LiDAR, forêt, parcellaire, structure agraire, Lorraine.

KEYWORDS. LiDAR, forest, field system, agrarian remains, Lorraine.

\section{Introduction}

Les structures agraires et parcellaires sont parmi les vestiges les plus fréquemment mis en évidence en forêt par les télédétections LiDAR aéroportées. Conservées sous forme de micro-reliefs, elles ont été préservées de l'érosion par le couvert végétal après leur abandon. Le LiDAR permet leur détection sur des surfaces considérables de plusieurs centaines à plusieurs milliers de kilomètres carrés. Il rend aussi possible une approche sur leur morphologie en fournissant des données tridimensionnelles.

La variété des formes que prennent ces vestiges n'est pas sans soulever des questions sur leur origine et leur fonction. Avec la multiplication des levés LiDAR depuis les années 2000, la nécessité de mettre en place des référentiels d'interprétation est assez vite apparue (par exemple East Sussex County Council 2012). Ce thème a ainsi été abordé lors d'un séminaire conjoint organisé dans le cadre 
de deux programmes de recherche, le programme « Rurland ${ }^{1} »$ et le programme «ODIT-CHEF ${ }^{2}$ » sur $^{-}$ «LiDAR et façons culturales» tenu en mai 2015 à Besançon. À cette occasion a été réalisée une typologie des vestiges agraires et parcellaires conservés sous forêt en Lorraine et ayant bénéficié d'acquisitions par LiDAR aéroporté. Cette typologie fait l'objet du présent article.

Pour l'élaborer, nous avons choisi d'aborder ces vestiges en trois étapes : 1) identification des formes élémentaires, notamment telles qu'elles apparaissent sur les visualisations LiDAR; 2) observations au sol et sondages afin de mieux appréhender l'origine de formation des vestiges, leur structure et leur taphonomie ; 3) analyse morphologique des organisations parcellaires. Nous avons complété cette étude morphologique et fonctionnelle par une approche chronologique. Celle-ci reste délicate, car ces structures sont difficiles à dater en elles-mêmes et il est nécessaire de recourir à plusieurs approches croisées pour y parvenir (analyses régressives des cartes et plans anciens, étude d'archives, fouille, connexion avec l'habitat, morphologie).

\section{Zones d'étude et méthodes}

Cette typologie se base sur les données provenant de quatre missions LiDAR (fig. 1, tabl. I). La première a été réalisée en mars 2007 au-dessus du massif forestier de Haye, localisé à côté de Nancy sur le revers de la côte de Moselle, dans le cadre d'un programme de recherche sur le plateau de Haye associant archéologues, écologues et forestiers (Georges-Leroy et al. 2012a). Cette mission a été complétée par un levé réalisé en mars 2009 par l'Institut géographique national (IGN) sur les forêts situées sur le plateau de Haye, entre le massif forestier de Haye et celui de Saint-Amond. De nombreux parcellaires anciens sont connus dans ce secteur, notamment dans les forêts de Thuilley-aux-Groseilles, Allain et Saint-Amond, même s'ils n'ont été que partiellement survolés.

\begin{tabular}{|c|c|c|c|c|c|c|c|c|}
\hline Secteur & Commanditaire & Date de vol & $\begin{array}{c}\text { Surface } \\
\text { (dont } \\
\text { forêt) } \\
\left(\mathrm{km}^{2}\right)\end{array}$ & $\begin{array}{c}\text { Hauteur } \\
\text { de vol } \\
\text { (m) }\end{array}$ & $\begin{array}{c}\text { Angle } \\
\text { de scan } \\
\left(^{\circ}\right)\end{array}$ & $\begin{array}{c}\text { Densité } \\
\text { moy. } \\
\text { émise } \\
\left(\mathrm{pts} / \mathrm{m}^{2}\right)\end{array}$ & $\begin{array}{c}\text { Densité } \\
\text { moy. } \\
\text { points sol } \\
\left(\mathrm{pts} / \mathrm{m}^{2}\right)\end{array}$ & $\begin{array}{l}\text { Résolution } \\
\text { MNT (m) }\end{array}$ \\
\hline $\begin{array}{l}\text { Forêt de Haye } \\
\qquad(54)\end{array}$ & $\begin{array}{c}\text { DRAC } \\
\text { Lorraine-INRA } \\
\text { Nancy-ONF }\end{array}$ & Mars 2007 & $\begin{array}{c}116 \\
(111)\end{array}$ & 700 & $+/-17$ & 11,3 & 2,11 & 0,50 \\
\hline $\begin{array}{c}\text { LGV Est - } \\
\text { partie } \\
\text { mosellane (57) }\end{array}$ & RFF & $\begin{array}{c}\text { Février-mars } \\
2008\end{array}$ & $102(22)$ & I & l & 8 & $\begin{array}{c}\text { 2,4 (forêt) } \\
\text { 3,9 (hors } \\
\text { forêt) }\end{array}$ & 0,25 \\
\hline $\begin{array}{l}\text { Plateau de } \\
\text { Haye (54) }\end{array}$ & IGN & Mars 2009 & $175(78)$ & 1200 & $+/-18$ & 5,5 & 1,45 & 1 \\
\hline Zone OPE (55) & ANDRA & $\begin{array}{l}\text { Décembre } \\
\text { 2009-mars } \\
2010\end{array}$ & $237(82)$ & 600 & $+/-20$ & 14 & 6,22 & 0,50 \\
\hline
\end{tabular}

Tableau I. Caractéristiques des levés LiDAR analysés

\footnotetext{
${ }^{1}$ « Rural landscape in north-eastern roman Gaul from the late La Tène period to the late Antiquity », 2014-2018, École Pratique des Hautes Études, Paris, programme de l’European Research Council, dirigé par Michel Reddé.

${ }^{2}$ Chantier «Construction historique des espaces forestiers (CHEF) », dirigé par Laure Nuninger, du programme « Observatoire des dynamiques industrielles et territoriales (ODIT) », 2011-2015, Maison des Sciences de l’Homme et de l'Environnement C. N. Ledoux, Besançon. 


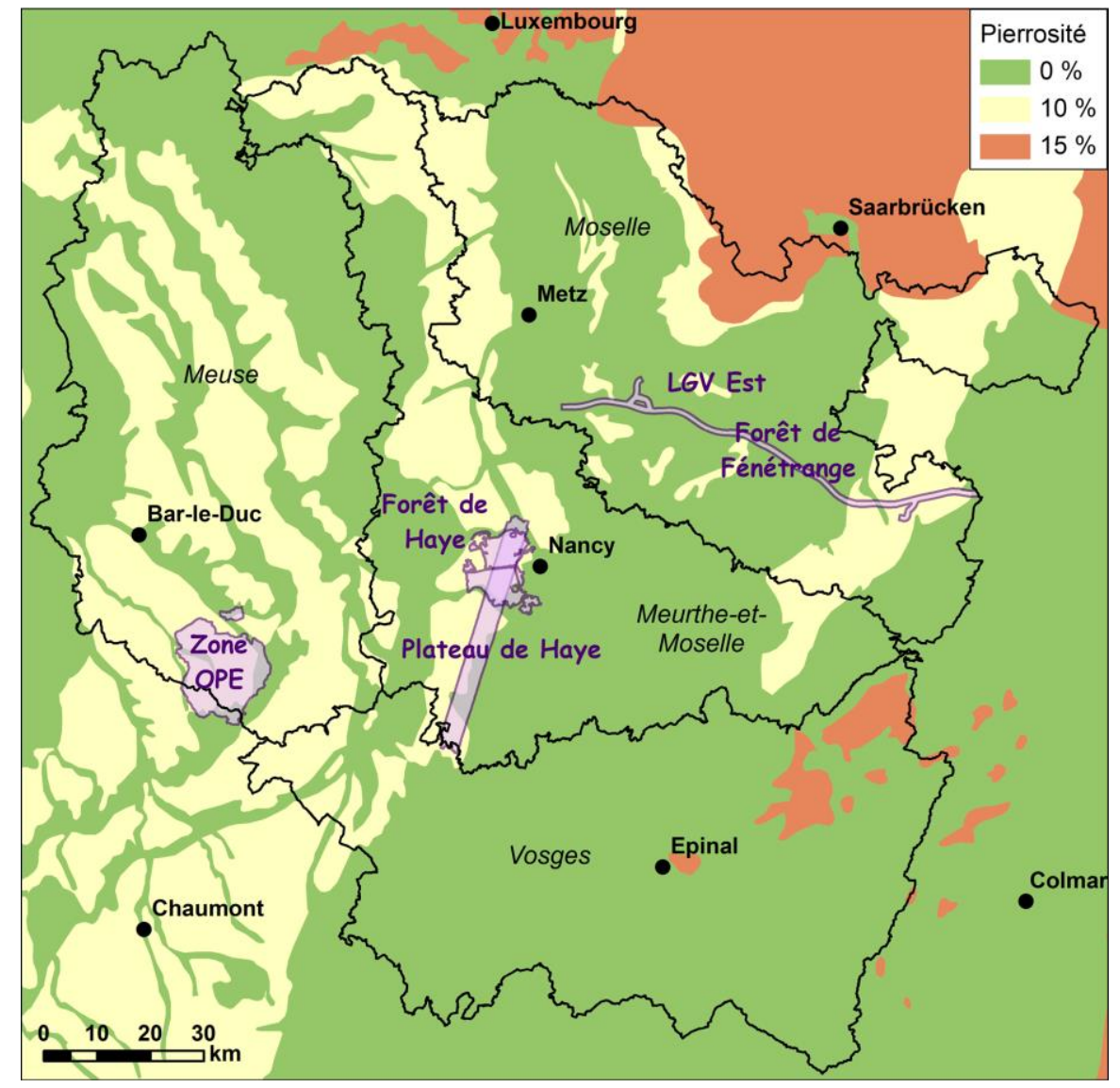

Figure 1. Localisation des levés LiDAR analysés, figurés en violet (SIG/DAO MGL; fond cartographique : The European Soil Database distribution version 2.0, European Commission and the European Soil Bureau Network, CD-ROM, EUR 19945 EN, 2004)

Durant l'hiver 2009-2010, un vol LiDAR a été réalisé à la demande de l'Andra, pour le projet d'Observatoire pérenne de l'environnement (OPE) mis en place autour d'un centre d'enfouissement des déchets radioactifs dans le sud de la Meuse. Il a fait l'objet d'une analyse archéologique et géomorphologique dans le cadre d'un partenariat entre l'Andra, la DRAC de Lorraine et l'université de Nancy 2.

Enfin, un levé a été commandité par Réseau ferré de France (RFF) lors de la réalisation de la deuxième phase de construction de la ligne à grande vitesse (LGV) Est entre Paris et Strasbourg (Georges-Leroy \& Viller 2016). Une bande d'un kilomètre de large a été survolée en mars 2008 sur le futur tracé entre Baudrecourt en Moselle et Vendenheim dans la banlieue de Strasbourg. Les analyses ont notamment porté sur le secteur du massif forestier de Fénétrange, étudié dans le cadre d'une thèse sur les mardelles forestières (Étienne et al. 2011, 2013). Le secteur le plus à l'est, qui traverse le Piémont vosgien, où de nombreux parcellaires sous forêt sont aussi inventoriés, n'a pas été utilisé ici.

Au total, ce sont environ $300 \mathrm{~km}^{2}$ de levés LiDAR en milieu forestier qui ont ainsi pu être mobilisés pour l'établissement de cette typologie. Les zones étudiées se localisent principalement sur des sols calcaires pierreux, en général peu épais. Seul le massif forestier de Fénétrange (LGV Est) recouvre des terrains marneux et limoneux. Ces sols ont bien sûr conditionné en partie les formes agraires qui s'y sont développées.

La méthode du multi-écho a été utilisée pour tous les levés, à l'exception de celui qui porte sur le sud meusien pour lequel a été mis en œuvre le full-waveform. Ils ont tous été réalisés lors du repos végétatif entre décembre et mars, avec des angles de scannage qui n'ont pas dépassé $+/-20^{\circ}$ pour une meilleure pénétration dans le couvert végétal. Les densités de points émises ont été assez importantes, variant entre 5,5 et $14 \mathrm{pts} / \mathrm{m}^{2}$. La densité moyenne des points sol après classification varie de 1,45 à $6,22 \mathrm{pts} / \mathrm{m}^{2}$, pour une résolution de MNT de $0,25 \mathrm{~m}$ à $1 \mathrm{~m}$. Les traitements de visualisation ont 
principalement consisté à utiliser des ombrages directionnels ou multi-directionnels, très ou moyennement rasants (élévation entre $3^{\circ}$ et $35^{\circ}$ ). D'autres traitements d'illumination ont été ponctuellement réalisés, notamment le Sky-view factor (Kokalj et al. 2011). L'approche morphologique s'est faite par l'exécution de profils en travers des structures, mais surtout par la superposition des courbes de niveaux, espacées entre 5 et $15 \mathrm{~cm}$, et des ombrages (Georges-Leroy et al. 2012a).

\section{Formes et origines des structures agraires et parcellaires}

\section{Les formes élémentaires observables sur les visualisations LiDAR (fig. 2)}
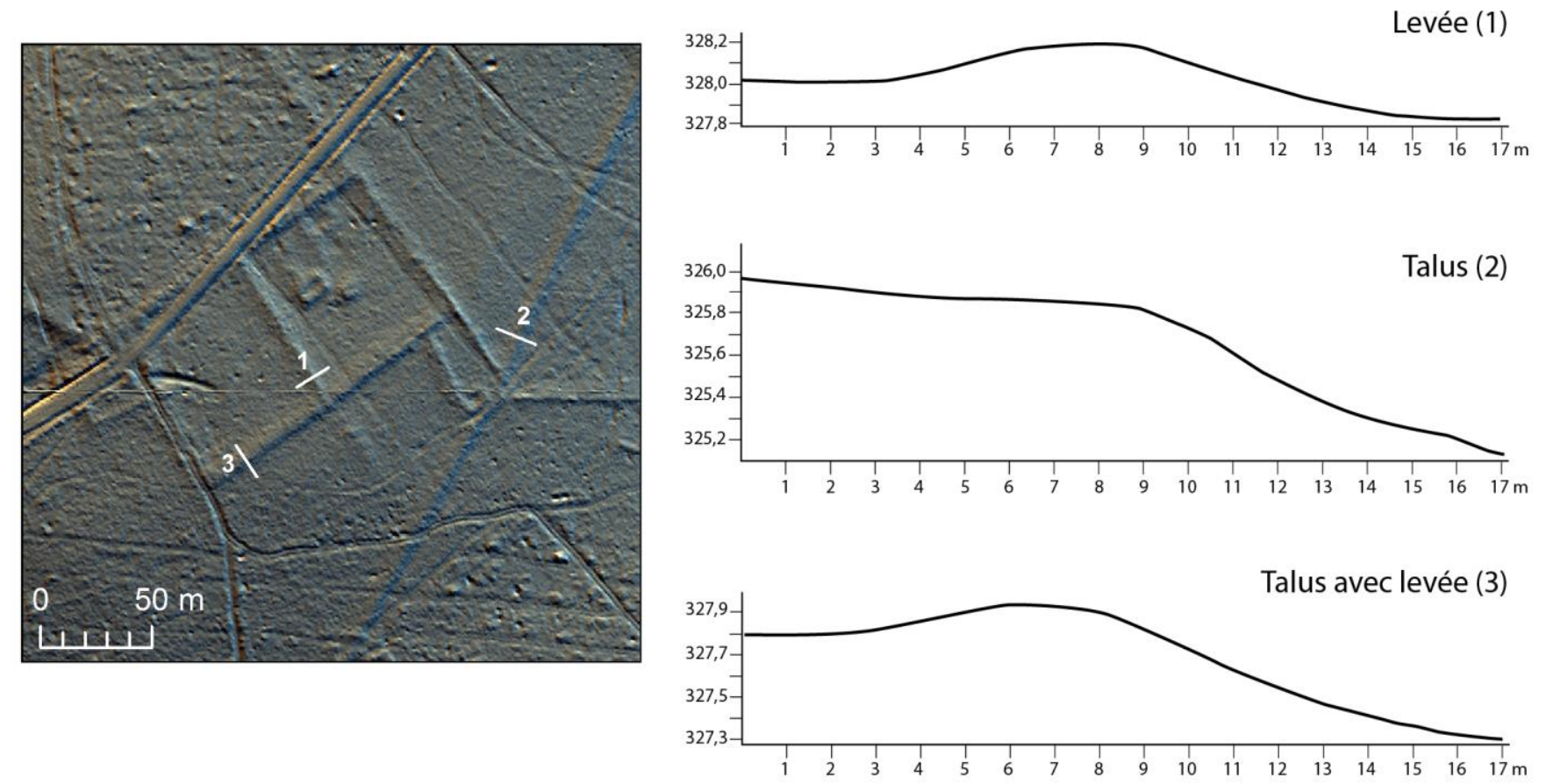

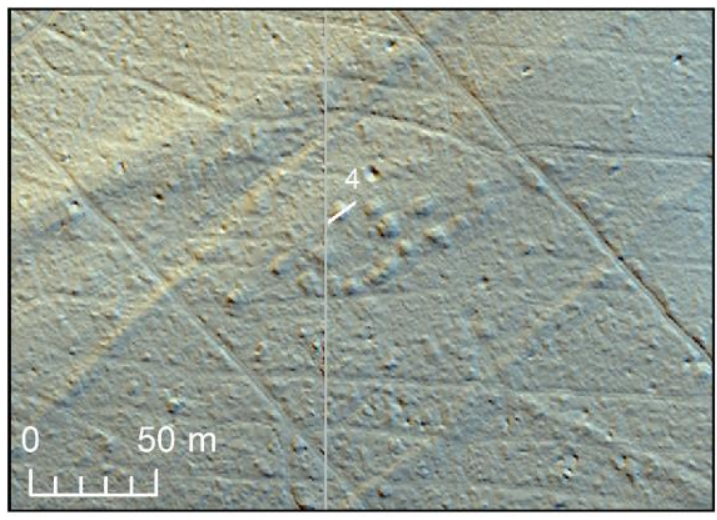

Tas (4)

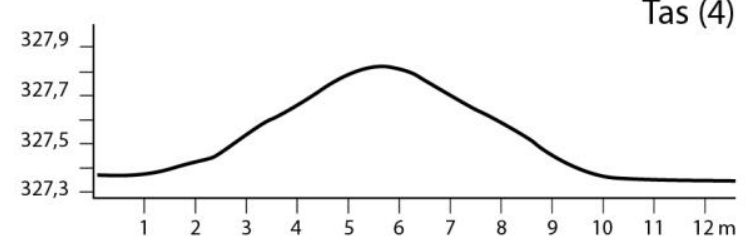

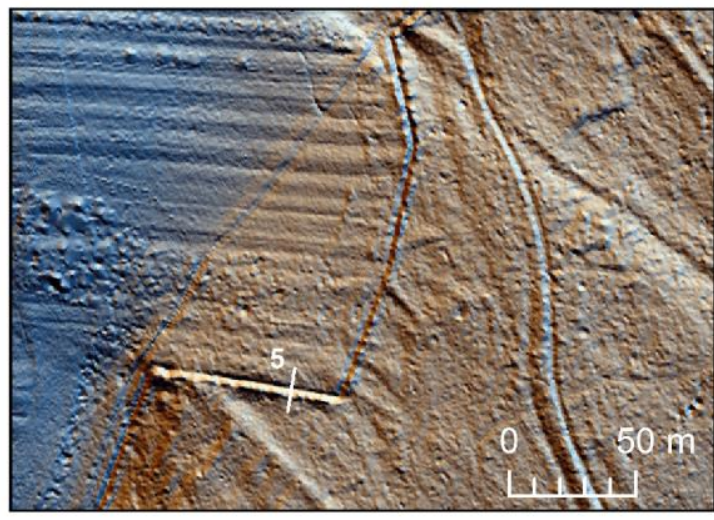

Levée et fossé (woodbank) (5)

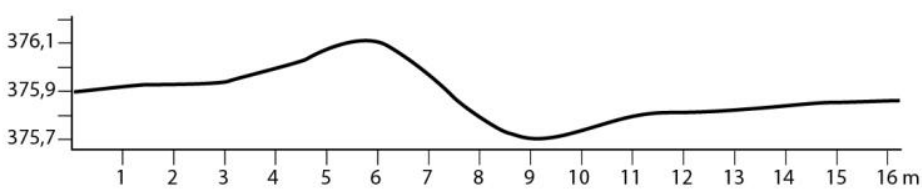

Figure 2. Formes élémentaires observables sur les visualisations LiDAR (SIG/DAO MGL; données LiDAR Haye 2007, DRAC Lorraine-INRA Nancy-ONF) 
Les formes élémentaires observables sur les visualisations LiDAR sont de quatre types, certains pouvant être associés : tas, levée, talus et fossé. Les tas d'origine agraire se présentent au sol comme des amas de pierres, ce qui est confirmé par des sondages en forêt de Haye (Georges-Leroy et al. 2012b). Hormis une taille souvent plus petite, ces tas de pierres apparaissent, sur les visualisations, de manière identique aux structures funéraires de type tumulus. Les levées prennent plusieurs formes au sol : des murs effondrés; de longs pierriers linéaires très étalés, aussi nommés murées, plus ou moins colmatés par la terre, dont certains étaient des murs en pierres sèches à l'origine; des levées de terre ; des champs bombés. Les talus, simples ou avec une levée, correspondent à des talus terreux pouvant être surmontés d'une murée. En forêt de Haye, les sondages à la tarière ou à la pelle mécanique ont montré qu'il peut s'agir soit de talus composés uniquement de terre, soit de talus recelant un pierrier interne.

- La dernière catégorie correspond à des fossés, associés à des levées de terre ${ }^{3}$.

Si ces vestiges découlent souvent d'une pratique agraire, ils peuvent en même temps délimiter des espaces, que cela soit volontaire (édification d'un muret) ou non (la pratique agraire produisant une structure de bord de parcelle). En cela ils constituent aussi des limites parcellaires. Cette dualité dans leur origine et/ou leur fonction autorise des approches tant sur la structuration spatiale des espaces sur lesquels ils se développent que sur la mise en valeur agraire de ceux-ci (Georges-Leroy et al. à paraître). Leurs origines sont variées : structures de délimitation forestière, structures d'épierrement, murs d'enclos, traces de culture, «terrasses ».

\section{Des structures parcellaires liées à la forêt (fig. 3)}

a

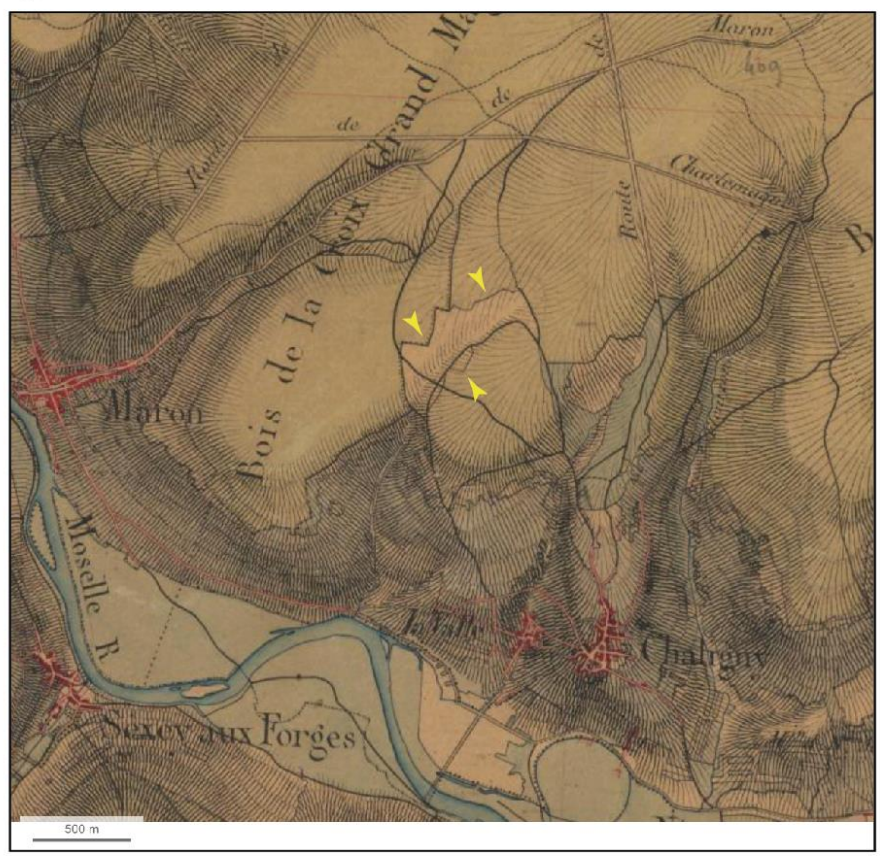

b

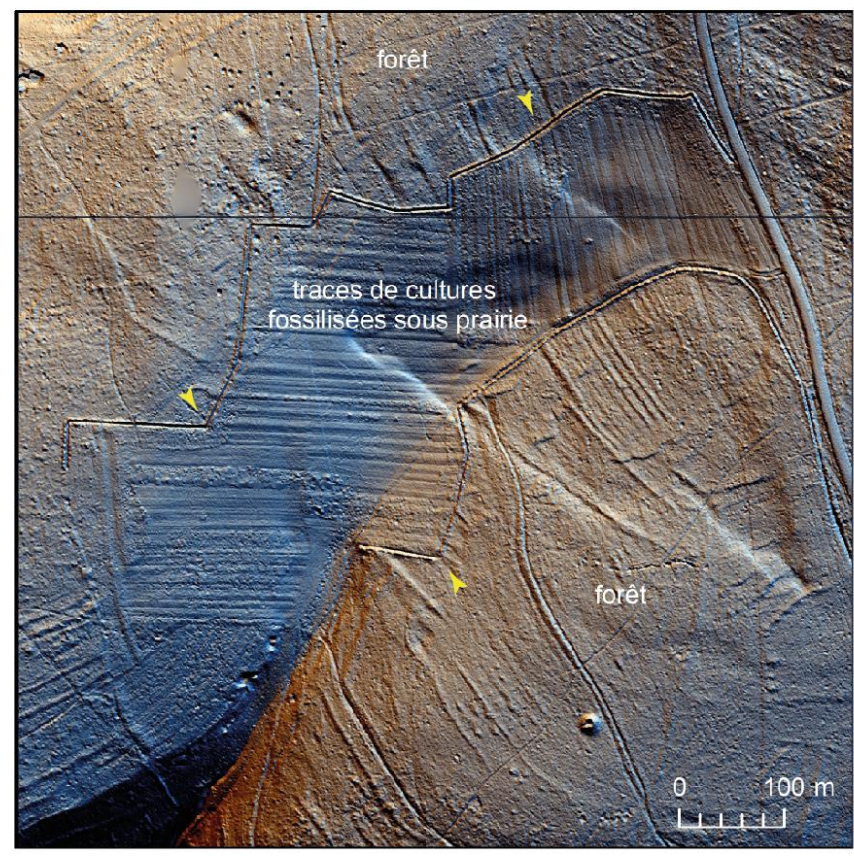

Figure 3. Limites forestières de type woodbank : a) carte de l'état-major, 1826-1833, feuille 69 (@ IGN), b) visualisation par ombrage multidirectionnel (16 directions, azimut $35^{\circ}$, exagération verticale 5 - données LiDAR Haye 2007, DRAC Lorraine-INRA Nancy-ONF)

D'anciennes limites forestières, nommées woodbank (Szabó 2010), se matérialisent sous la forme d'un talus de terre bordé d'un fossé, le talus étant situé côté forêt. Dans le massif de Haye et dans le sud meusien, où ces structures ont été systématiquement cartographiées, elles correspondent aux

\footnotetext{
${ }^{3}$ Des fossés encadrent aussi les buttes centrales des voies antiques qui ne sont pas abordées ici. Par ailleurs, les fossés isolés, présents 
limites des forêts représentées sur la carte d'état-major de 1826-1833, mais elles sont probablement antérieures au XIX ${ }^{\mathrm{e}} \mathrm{s}$.

\section{Des structures d'épierrement (fig. 4)}

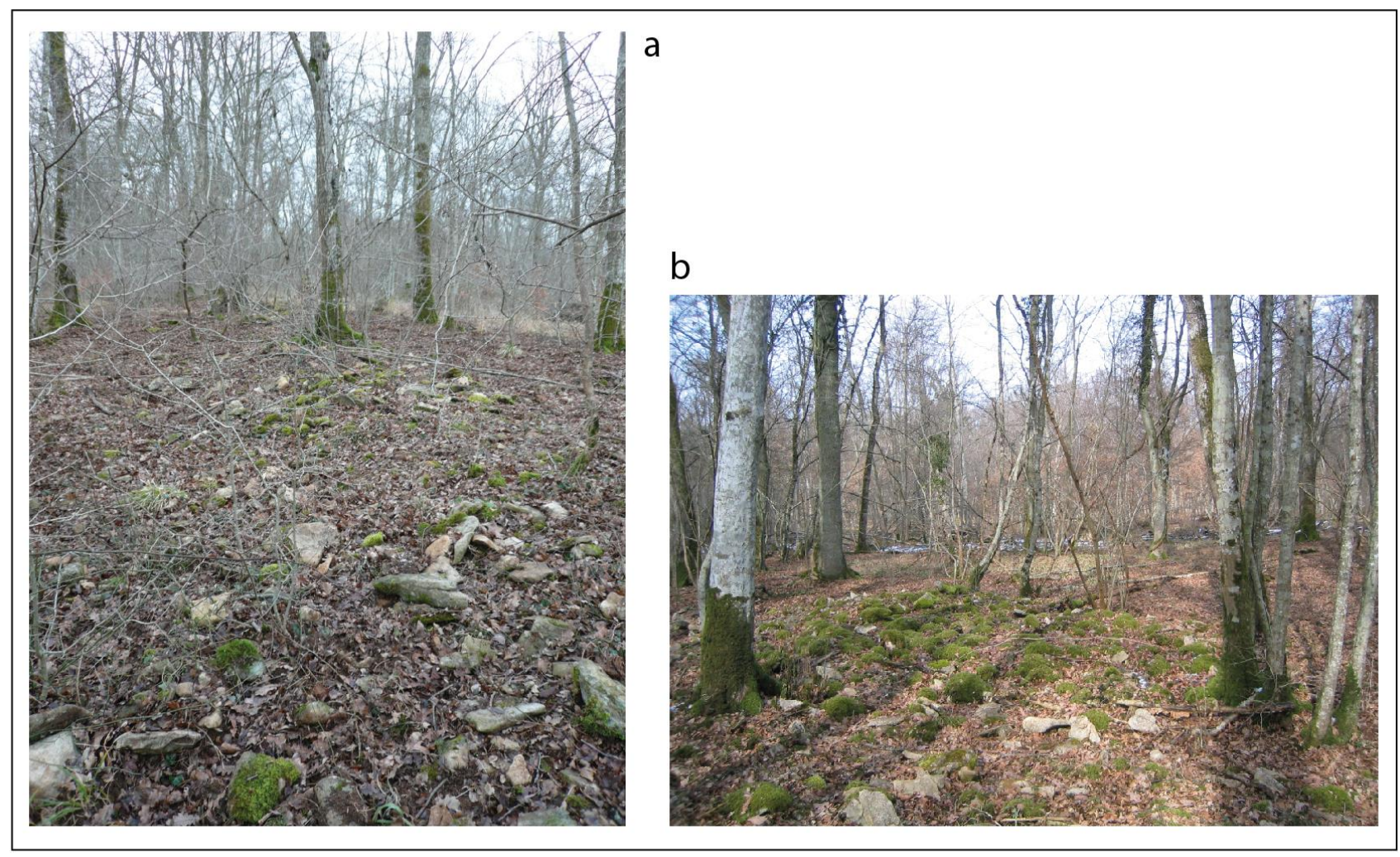

Figure 4. Structures d'épierrement en forêt de Haye : a) murée, b) tas (clichés MGL)

Tas et murées résultent de l'épierrement des champs, voire des prairies de fauche. Mais ces pierriers ont aussi servi à délimiter des enclos pour le bétail et à matérialiser pour certains de véritables limites parcellaires. La pratique de l'épierrement est attestée chez les agronomes latins comme Columelle (COL., R. R., II, 2 ; II, 17). De leur côté, les arpenteurs latins citent, parmi les types de limites ou de clôtures possibles, des murs de pierres, soit faits de pierres amoncelées sans construction, soit édifiés. D'autres évoquent aussi des amas de pierres utilisés comme bornes (Chouquer \& Favory 2001 : 188189). Philippe Blanchemanche énumère quant à lui les diverses modalités d'épierrement des terres aux $\mathrm{XVIII}^{\mathrm{e}}$ et XIX ${ }^{\mathrm{e}}$ s., parmi lesquelles l'édification de tas à l'extrémité ou au milieu des champs, ou celle de murgers, même s'il précise que la raison première de l'épierrement d'un champ ne consiste pas à le clôturer (Blanchemanche 1990 : 230-242). Les structures d'épierrement conservées en élévation, de type tas ou murée/murger, sont donc peu datables en elles-mêmes. Un degré de colmatage important des pierres par de la terre peut toutefois suggérer une certaine ancienneté, mais sans plus de précision. Ainsi, le tas d'épierrement antique fouillé à Gondreville, en forêt de Haye se présentait comme un amas de pierres très colmatées.

\section{Des murs d'enclos}

Certaines des murées antiques étaient à l'origine de véritables murs en pierres sèches, comme ceux mis en évidence en forêt de Haye, ou en pierres liées à l'argile, comme ceux d'un enclos d'habitat fouillé à Thuilley-aux-Groseilles ${ }^{4}$. À Frouard, le mur maçonné du parc Lattier, réserve de chasse du $\mathrm{XVII}{ }^{\mathrm{e}}$ s., est aujourd'hui partiellement effondré et ressemble à une murée dans certains secteurs 
(fig. 5). D'autres murs d'enclos du XIX ${ }^{\mathrm{e}}$ s. plus ou moins effondrés ont aussi été identifiés dans d'autres secteurs de la forêt de Haye.

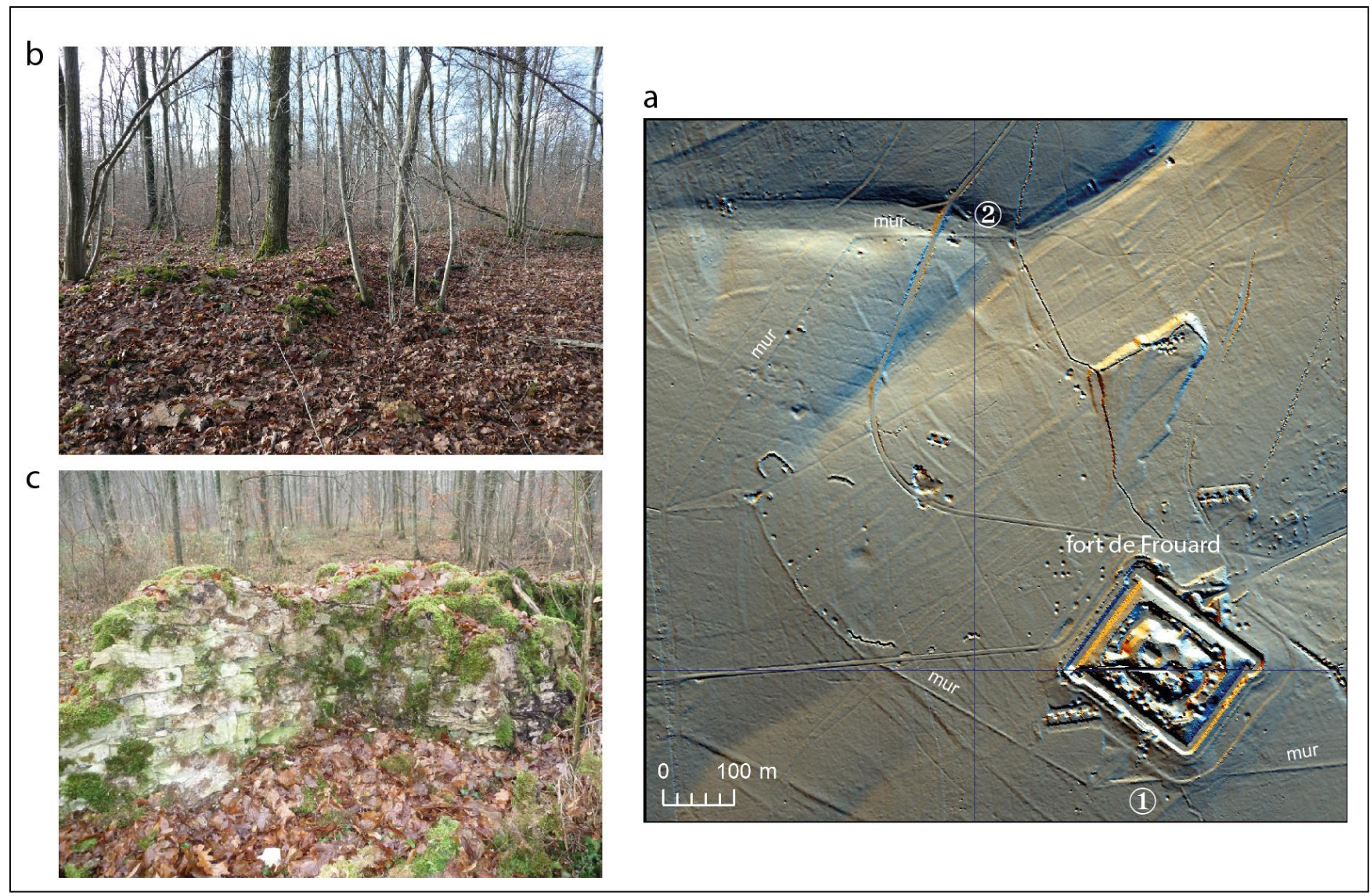

Figure 5. Parc Lattier (XVIII s.), réoccupé par le fort de Frouard (fin du XIX s.) : a) visualisation par ombrage multidirectionnel (16 dir., az. 10 - données LiDAR Haye 2007, DRAC Lorraine-INRA Nancy-ONF), b) vue du mur totalement ruiné (point 1), c) vue du mur partiellement ruiné (point 2) (clichés MGL)

\section{Des traces de culture}

De nombreux talus et levées résultent d'une mise en culture.

Ainsi, certains talus de terre correspondent à des rideaux de culture (fig. 6). Ces derniers sont des dénivellations très fortes qui séparent deux bandes de culture disposées perpendiculairement à l'axe de la pente. Ils sont obtenus par le labour qui établit progressivement et involontairement cette accumulation de terre vers le bas de la parcelle (Zadora-Rio 1991 : 173-174, Comet 1992 : 102-103). Certains de ces talus implantés dans des zones pentues appartiennent très probablement à cette catégorie. En revanche, d'autres, installés dans des zones plus planes, sont d'interprétation moins évidente. C'est notamment le cas des talus, hauts de quelques dizaines de centimètres, localisés dans la forêt communale de Frouard (fig. 6a, c) et dont la reconnaissance à la tarière a montré qu'il s'agissait de talus de terre. Leur hauteur et leur profil ne permettent pas d'y voir la trace d'une dérayure (rigole profonde séparant deux planches de culture). Nous proposons donc, malgré leur faible dénivelé, d'y voir tout de même des rideaux de culture. 
a. Forêt communale de Liverdun

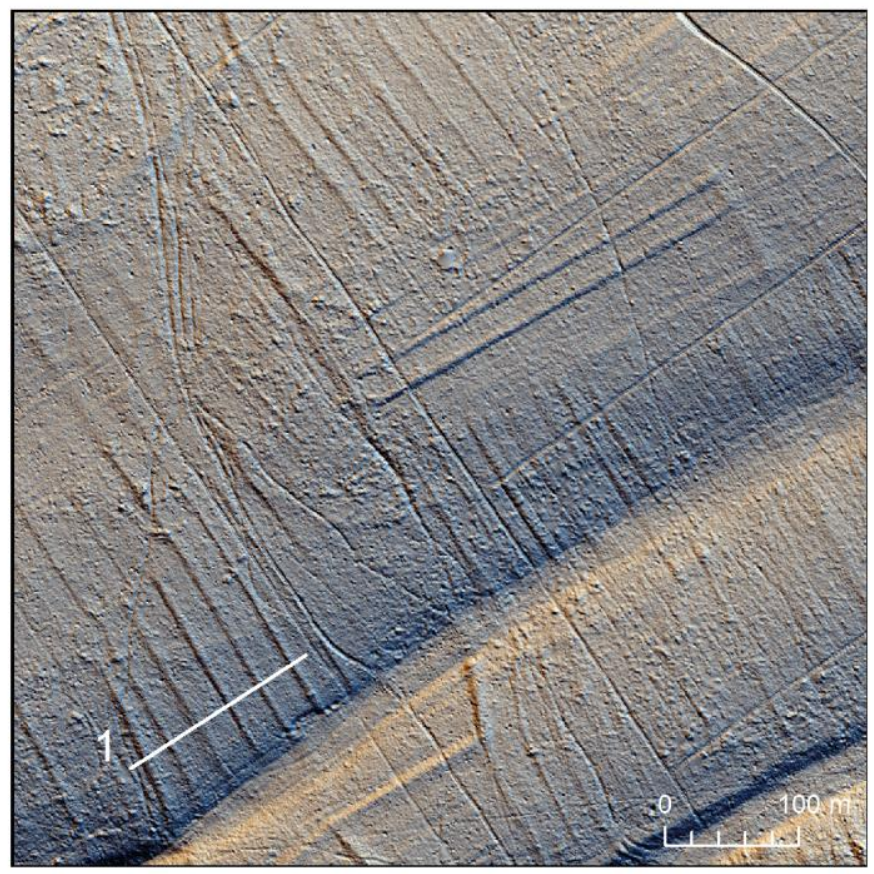

b. Forêt domaniale de Haye à Maron

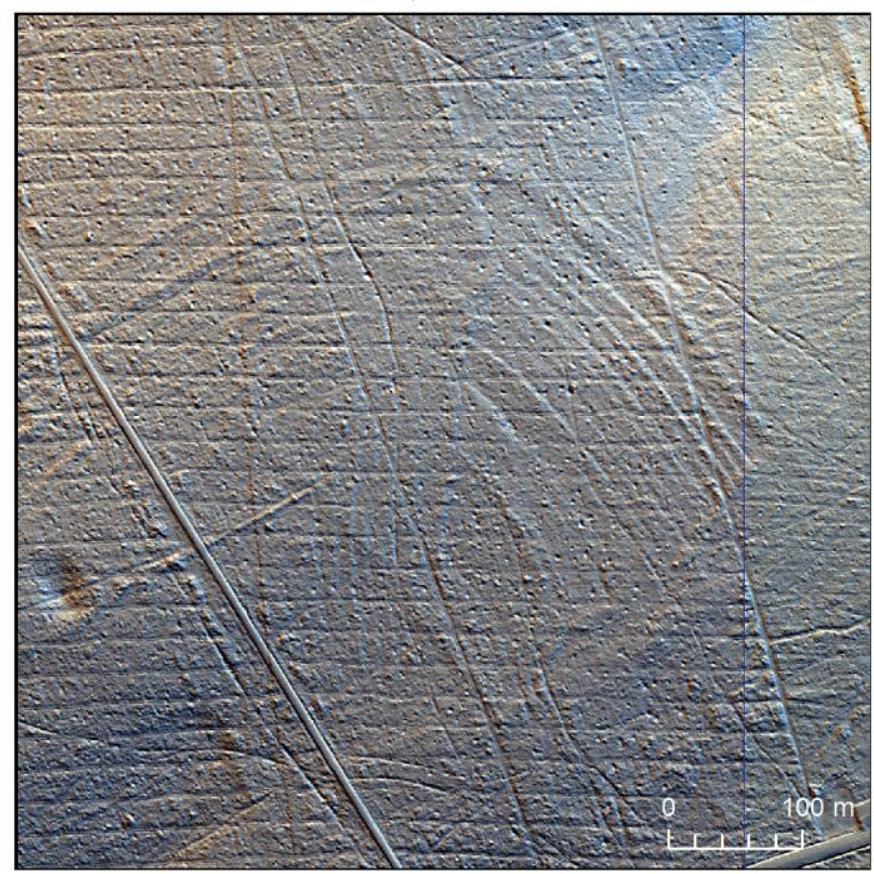

c. Profil 1

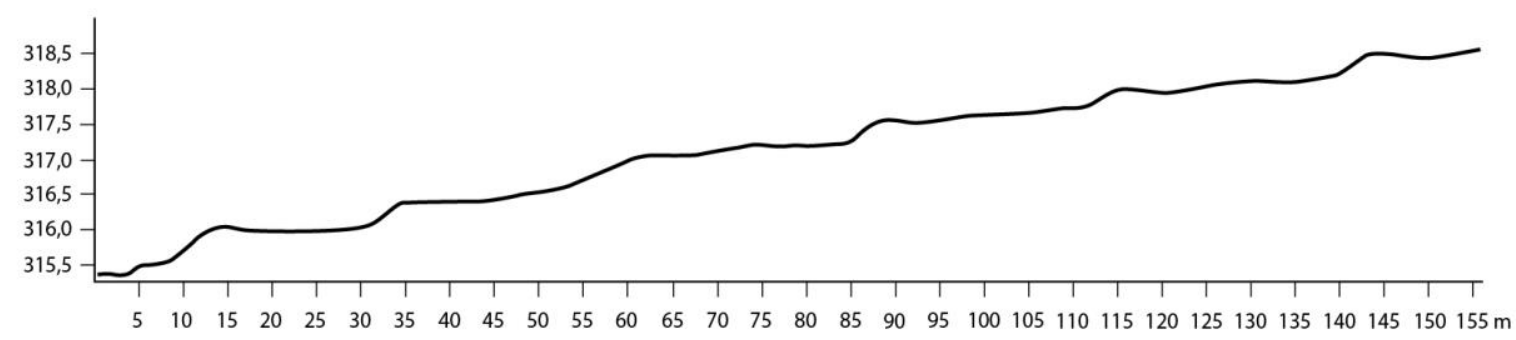

Figure 6. Rideaux de cultures en forêt de Haye : a, b) visualisations par ombrage multidirectionnel (16 dir., az. 35, ex. vert. 5- données LiDAR Haye 2007, DRAC Lorraine-INRA Nancy-ONF), c) profil 1

Des billons, nommés champs bombés en Lorraine, ont aussi été repérés dans les forêts du secteur. Se présentant sur les visualisations LiDAR comme des levées parallèles séparées par un creux, ils sont le résultat d'un labour en ados avec une charrue à versoir fixe qui fait un parcours en spirale depuis le centre jusqu'à la périphérie (Zadora-Rio 1991: 166-171, Blaising 2010: 9-10). Le bombement s'obtient progressivement en labourant toujours de la même façon un champ. Les bombements repérés en forêt de Haye sont parallèles, peu élevés $(10-20 \mathrm{~cm}$ ) et larges de 6 à $12 \mathrm{~m}$ (fig. 7a-d). Fréquents dans les zones de prairie du plateau de Haye (fig. 7e), les champs bombés sont peu nombreux dans les forêts objets du présent article. 

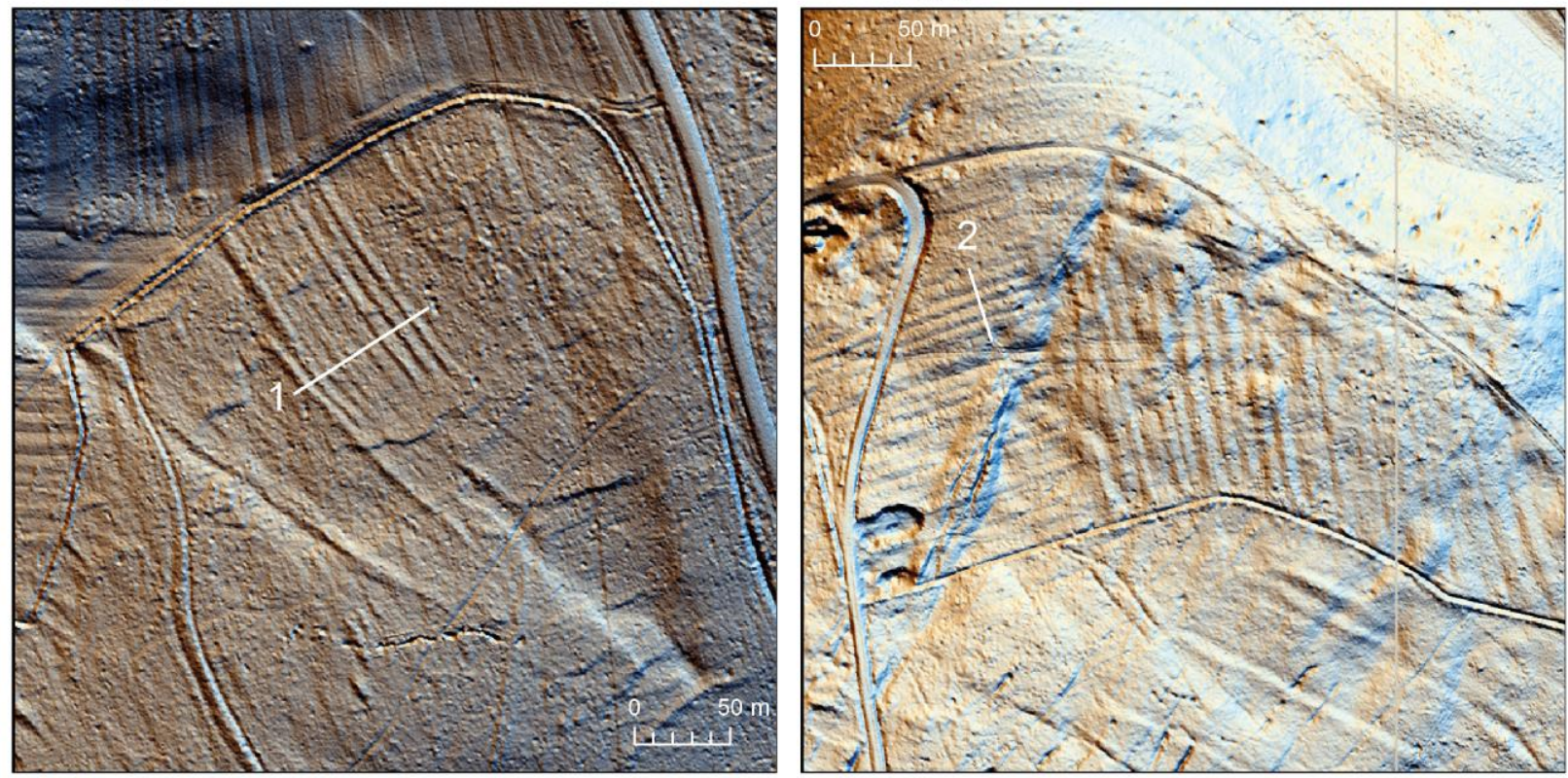

Visualisation par ombrage multidirectionnel ( 16 dir.), azimut $35^{\circ}$, exagération verticale 5 (données lidar Haye 2007, DRAC Lorraine-INRA Nancy-ONF)

e. Colombey-lès-Belles - Vézelise

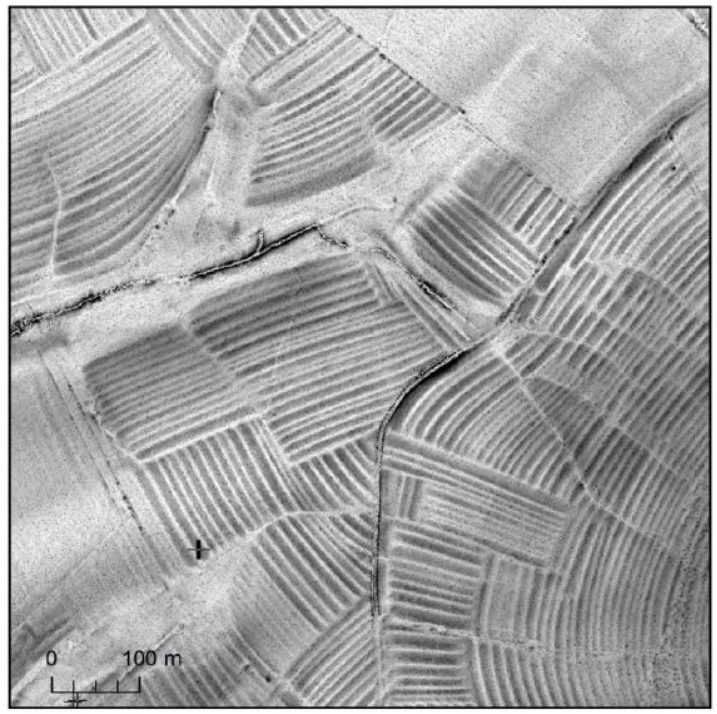

Visualisation par sky-view factor (4 directions, rayon 10, exagération verticale 2 (données lidar IGN 2009)

\section{Profil 1}

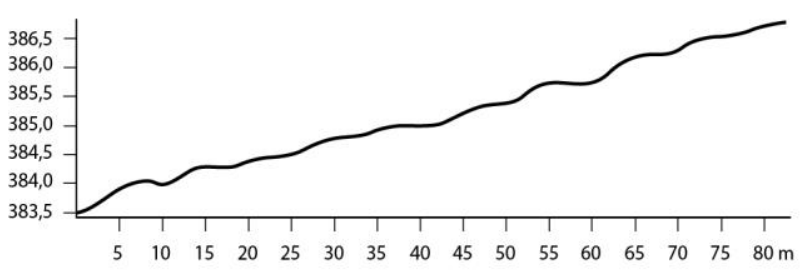

d. Profil 2

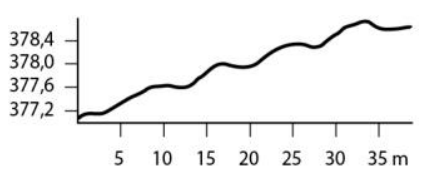

Figure 7. Champs bombés en forêt de Haye (a-d) et en prairie sur le plateau de Haye (e)

Des crêtes de labour potentielles ont également été identifiées. Rappelons que les crêtes de labour correspondent à un exhaussement, à l'extrémité des parcelles, dû à l'accumulation de la terre déplacée par le mouvement de la charrue dans le sens du labour et par les particules de terre qui tombent du soc et du versoir lorsque le laboureur fait tourner l'attelage en bout de champ (Callot, 1980, Zadora-Rio 1991 : 171-172). Un tel exemple a été observé à l'extrémité sud d'un groupe de champs en lanières en forêt de Haye à Chaligny (fig. 7a). Dans ce même massif forestier, dans le secteur de la Petite Haye, des bourrelets de terre parallèles, longs de 8-12 m et larges de quelques mètres, espacés d'autant, formant un talus discontinu, ont aussi été repérés sur les visualisations LiDAR (fig. 8). Ces vestiges pourraient correspondre à des crêtes de labour en cours de formation, ce qui expliquerait qu'elles ne soient pas continues. Des vestiges assez comparables sont connus en Angleterre, fossilisés par des prairies (Hall 1982 : 6-9). 

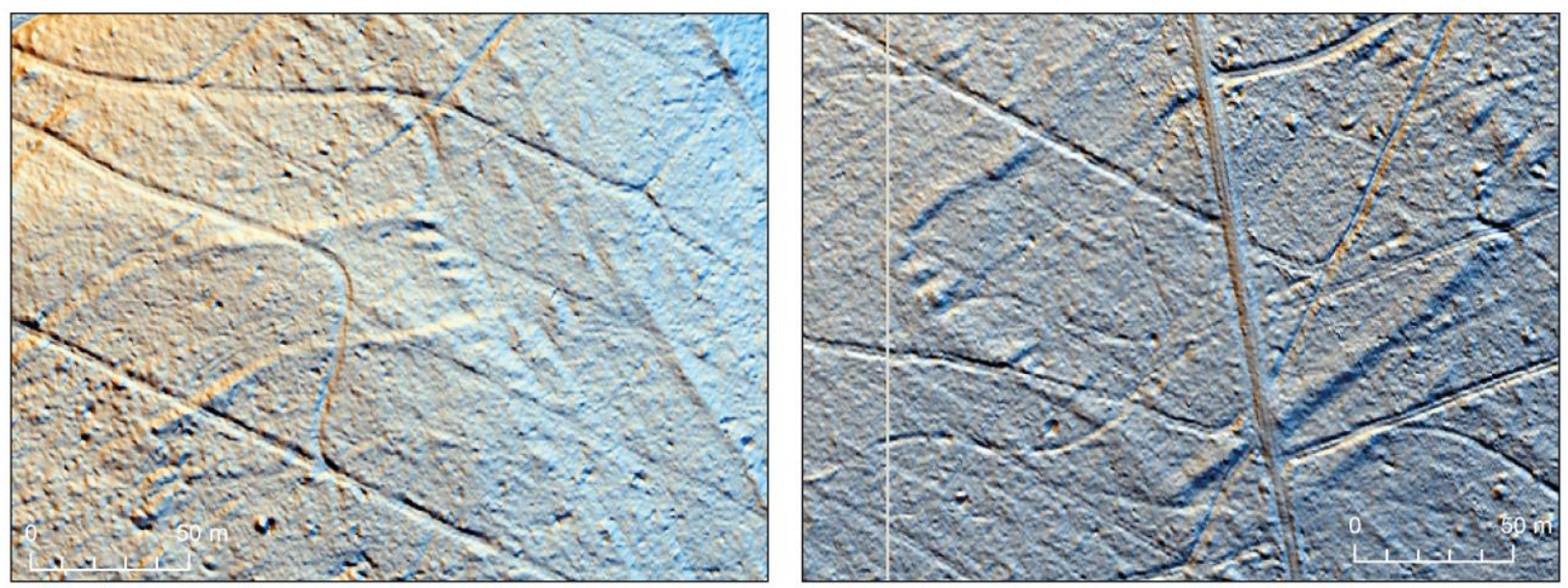

Figure 8. Crêtes de labour dans la Petite Haye, en forêt de Haye : visualisations par ombrage multidirectionnel (16 dir., az. 35, ex. vert. 5- données LiDAR Haye 2007, DRAC Lorraine-INRA Nancy-ONF)

Enfin, d'autres traces de labours sont peut-être visibles sur les images LiDAR, mais leur interprétation est délicate. Par exemple, à Gondreville, dans un secteur où sont connus des établissements antiques, on observe de petits talus très peu marqués, distants de 17 à $20 \mathrm{~m}$, à l'intérieur d'une parcelle (fig. 9a) mais aussi des traces en creux recoupant les parcellaires antiques (fig. 9b).

a.

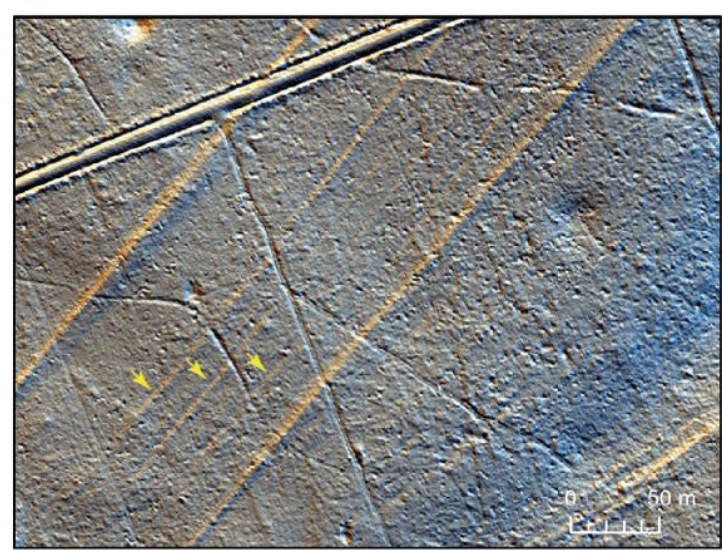

b.

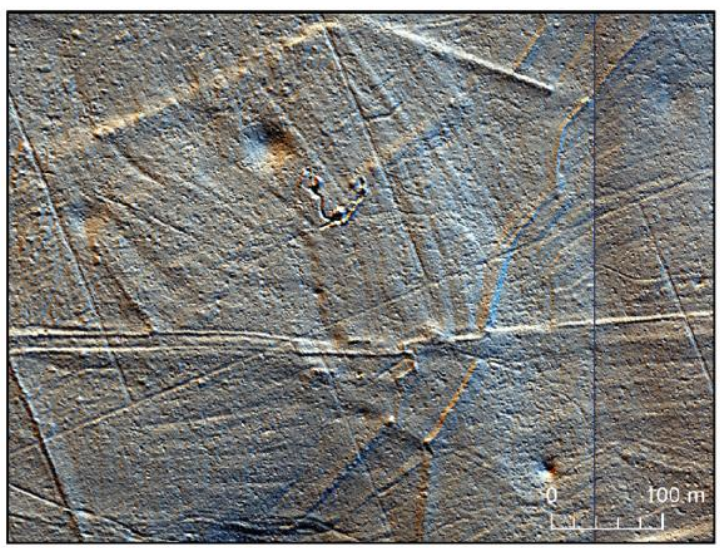

Figure 9. Traces potentielles de labours à Gondreville, en forêt de Haye : visualisations par ombrage multidirectionnel (16 dir., az. 10, ex. vert. 2 - données LiDAR Haye 2007, DRAC Lorraine-INRA Nancy-ONF)

Les traces de culture sont difficiles à dater de par leur simple morphologie. En effet, si la charrue est utilisée au Moyen Âge, certains chercheurs comme Pierre Ouzoulias envisagent une utilisation de versoirs sur les araires dès l'Antiquité, mais avec un usage limité, probablement réservé à des actions spécifiques (labours pour défricher ${ }^{5}$ ). Les outils utilisés aux deux périodes pourraient donc induire des formes agraires assez similaires. Ainsi, les billons, surtout les plus marqués, semblent typiques d'une utilisation de la charrue, mais G. Haudricourt et M. Jean-Brunhes Delamare (2000) évoquent des exemples de bombement central d'un champ obtenu avec un araire ou une houe. Quoi qu'il en soit, les exemples les plus anciens de champs bombés connus par l'archéologie en Lorraine sont datés du IX ${ }^{\mathrm{e}} \mathrm{s}$. (Blaising 2010, Gérard 2012). Les crêtes de labour seraient quant à elles caractéristiques de la charrue (Haudricourt \& Jean-Brunhes Delamare 2000 : 445). 


\section{La question des «terrasses»}

Trois talus, datés de l'époque romaine, ont fait l'objet de sondages archéologiques en forêt de Haye en 2005. Les coupes en travers réalisées à cette occasion permettent de mieux comprendre leur formation (Georges-Leroy et al. 2012b) (fig. 10). Elles ont révélé la présence au cœur du talus d'un muret en pierres sèches, large de $0,60 \mathrm{~m}$ à $1,10 \mathrm{~m}$, conservé sur $0,30 \mathrm{~m}$ environ de hauteur. À l'origine, on peut estimer que ces pierriers devaient s'élever entre $0,40 \mathrm{~m}$ et 0,70 m de hauteur, d'après la partie effondrée. On observe une accumulation de limons en amont des pierriers et le dégagement en aval de la dalle calcaire qui apparaît immédiatement sous l'humus forestier. Les analyses micromorphologiques en amont et en aval du talus attestent d'une mise à nu et d'un remaniement du sol. Cette mise à nu des sols a dû entraîner un colluvionnement de la terre provenant de la partie haute de la parcelle et son blocage par le pierrier en aval. L'absence de pierres dans ce niveau révèle un épierrement systématique probable, rehaussant le pierrier avec le temps. La présence de pierriers sur d'autres talus doit ainsi témoigner de la poursuite ou d'une reprise de l'épierrement. Ces «terrasses » semblent donc résulter davantage d'une mise en culture que d'une construction volontaire avec apport de matériaux, par exemple pour augmenter l'épaisseur de sol en amont du talus. La grande variabilité morphologique pour un même linéament, telle une murée prolongeant un talus, va aussi dans ce sens. On est donc en présence de structures qui se situent entre la terrasse construite et le rideau de culture.

a. Vue de la coupe au niveau du pierrier

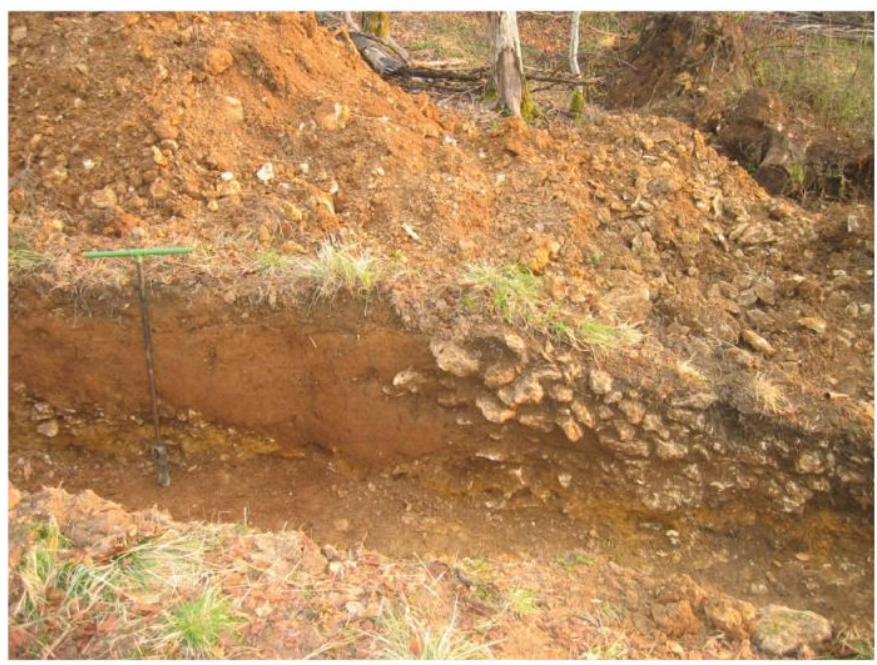

Cliché : Étienne Dambrine, avril 2005

b. Coupe ouest-est c. Proposition de restitution

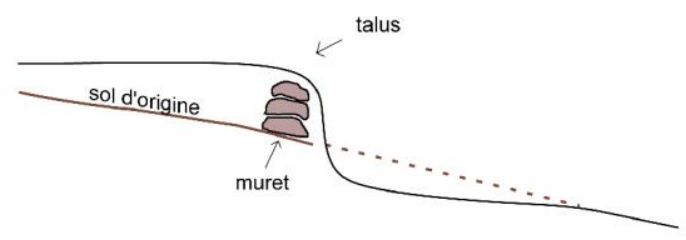

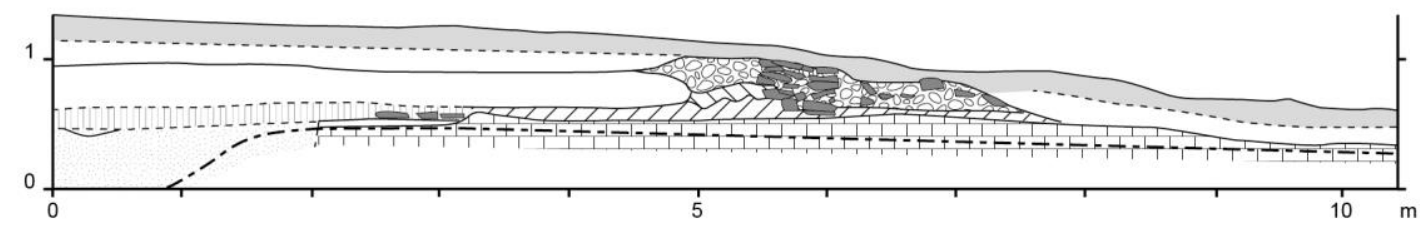

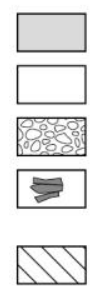

Humus limoneux beige gris (sol forestier actuel) Limon brun beige ou limon argileux brun ocre Pierres calcaires formant un pierrier lâche et destructuré Gros blocs de dalle calcaire (talus 1) ou muret en pierres sèches

Poche d'argile brune

Relevé : Jean-Denis Laffite, avril 2005
Argile rouge de décalcification des plaquettes calcaires (terra fusca)

Plaquettes calcaires mêlées d'argile rouge

Dalle calcaire

Sable rouge compact (apport fluviatile) colmatant une faille

Figure 10. Talus/terrasse de Maron, en forêt de Haye : a, b) sondage d'avril 2005, c) proposition de restitution 


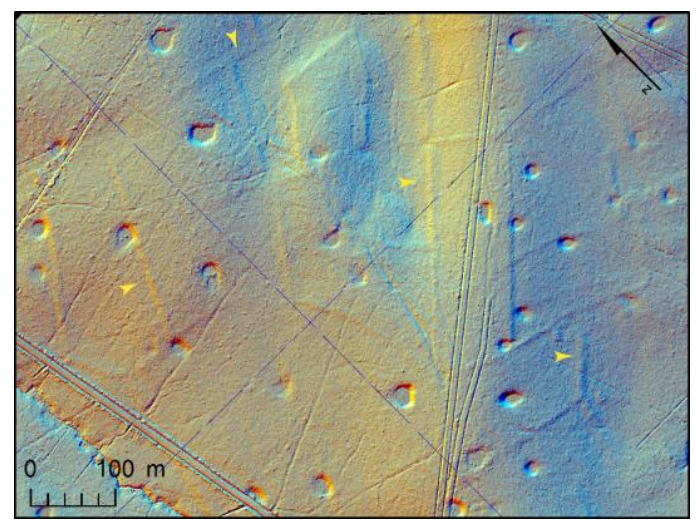

Figure 11. Talus de terre (flèches jaunes) et mardelles (dépressions humides) dans la forêt domaniale de Fénétrange, visualisation par ombrage multidirectionnel (16 dir., az. 10-données LiDAR RFF)

Parmi les talus uniquement constitués de terre, ceux qui ont été sondés lors des diagnostics archéologiques sur la LGV Est par l'Inrap, en 2008, en forêt de Fénétrange (Georges-Leroy \& Viller 2016) ont une origine qui nous échappe pour l'instant. Quasi invisibles au sol, ils sont impossibles à détecter sans un levé LiDAR. Il est difficile d'y voir uniquement le résultat d'une mise en culture du fait de leur organisation (ils délimitent des parcellaires à trame polygonale - cf. infra). S'agit-il d'une édification volontaire et dans ce cas de structures parcellaires uniquement?

\section{Morphologie des parcellaires}

L'analyse morphologique des parcellaires contribue aussi à la compréhension de ces structures agraires/parcellaires. Plusieurs morphologies de parcellaires ont ainsi été mises en évidence dans les forêts lorraines.

\section{Des parcellaires à trame polygonale ou en bandes longitudinales (fig. 12)}

a. Forêt de Haye (partie sud-ouest)

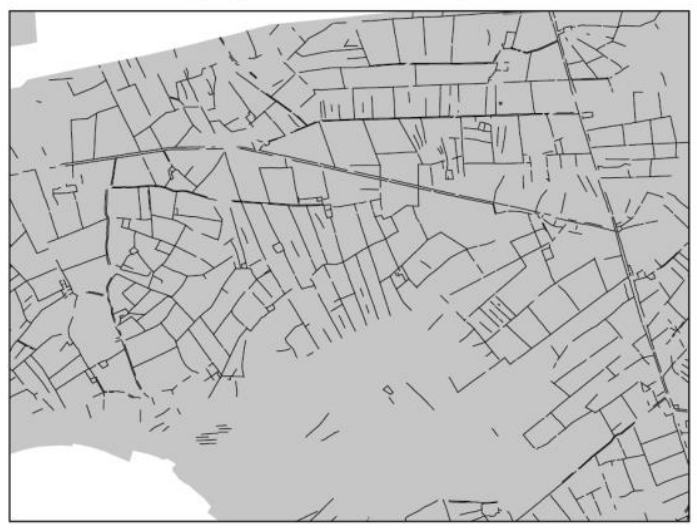

b. Forêt de Fénétrange

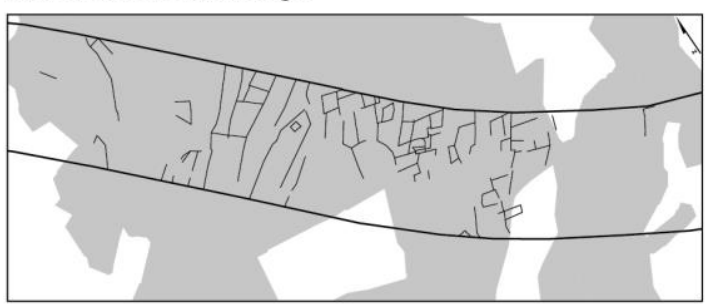

c. Forêt de Saint-Amond (partie nord)

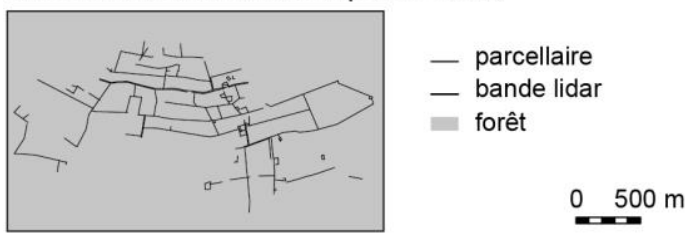

Figure 12. Parcellaires à trame polygonale et à bandes coaxiales (forêts de Haye, de Saint-Amond et de Fénétrange) (SIG/DAO MGL; données archéologiques : M. Georges-Leroy, David Étienne, INRA Nancy)

De nombreux ensembles appartiennent à la catégorie des parcellaires non limités dans lesquels Gérard Chouquer distingue deux types : les réseaux à trame polygonale et à trame en bandes coaxiales (Chouquer 2009 : 249). Les parcellaires de la forêt de Haye appartiennent plutôt à la catégorie des parcellaires à trame polygonale, mais une organisation en bandes longitudinales est peut-être reconnaissable dans certains secteurs, tout comme dans la partie nord de celui de Saint-Amond ou à Allain, sur le plateau de Haye (Chouquer 2006 : 75, Favory 2011 : 405). 
Lorsqu'ils sont localisés sur des sols pierreux, qui ont nécessité un épierrement pour être cultivés, ces parcellaires se matérialisent par des murées et des talus (simples ou surmontés de murées). Les exemples bien étudiés sont antiques comme ceux de la forêt de Haye, de Thuilley-aux-Groseilles, d'Allain ou de Saint-Amond, principalement datés grâce aux fermes connectées au parcellaire. Ils délimitent des espaces de surfaces parfois importantes, jusqu'à 15-16 ha en forêt de Haye pour une surface moyenne de 4 ha (Georges-Leroy et al. à paraître).

Dans les zones non pierreuses, ces parcellaires à trame polygonale sont composés de talus terreux, comme dans la forêt de Fénétrange. Dans ce cas, une datation gauloise ou antique est envisageable : les analyses palynologiques réalisées sur les mardelles tourbeuses localisées à proximité montrent en effet une ouverture du milieu durant l'Antiquité, puis une reforestation jusqu'à nos jours avec quelques rares défrichements durant le Moyen Âge (Étienne et al. 2013). Ces analyses, ainsi que des analyses ADN, suggèrent plutôt la présence de pâtures et de troupeaux durant l'Antiquité (Étienne et al. 2015).

\section{Des parcellaires laniérés, parfois croisés}

Deux types de parcellaires laniérés, parfois croisés, existent en forêt de Haye.

Le premier comprend des groupes de talus parallèles assez rapprochés (entre 7 et $30 \mathrm{~m}$ ), plus ou moins marqués, qui se présentent au sol comme des talus terreux, parfois légèrement bombés. Rectilignes ou courbes, en épousant les formes du relief, ils s'organisent en quartiers rarement délimités. Dans les zones pentues, les talus sont installés parallèlement à la pente et peuvent être assez rapprochés. L'ensemble le plus représentatif est conservé dans la forêt communale de Liverdun (fig. 13a). Plusieurs blocs de parcelles en lanières, longs de 150 à $650 \mathrm{~m}$ de long, sont constitués de talus distants de 10 à $30 \mathrm{~m}$ et hauts de 15 à $50 \mathrm{~cm}$. Installés en majorité dans le sens opposé à la pente, ils se développent perpendiculairement ou parallèlement les uns aux autres sur un espace d'environ 350 ha. Du fait de l'absence de connexion avec des habitats et d'éléments de datation intrinsèques, seules des fourchettes chronologiques assez imprécises peuvent être proposées. En résumé, ces structures agraires sont postérieures à l'époque romaine et au moins antérieures au XVIII ${ }^{\mathrm{e}}$ s., voire au $\mathrm{XVI}^{\mathrm{e}} \mathrm{s}$. pour certaines, d'après certains recoupements, mais aussi d'après l'analyse de cartes et textes anciens (Georges-Leroy \& Zeller-Belville 2018).

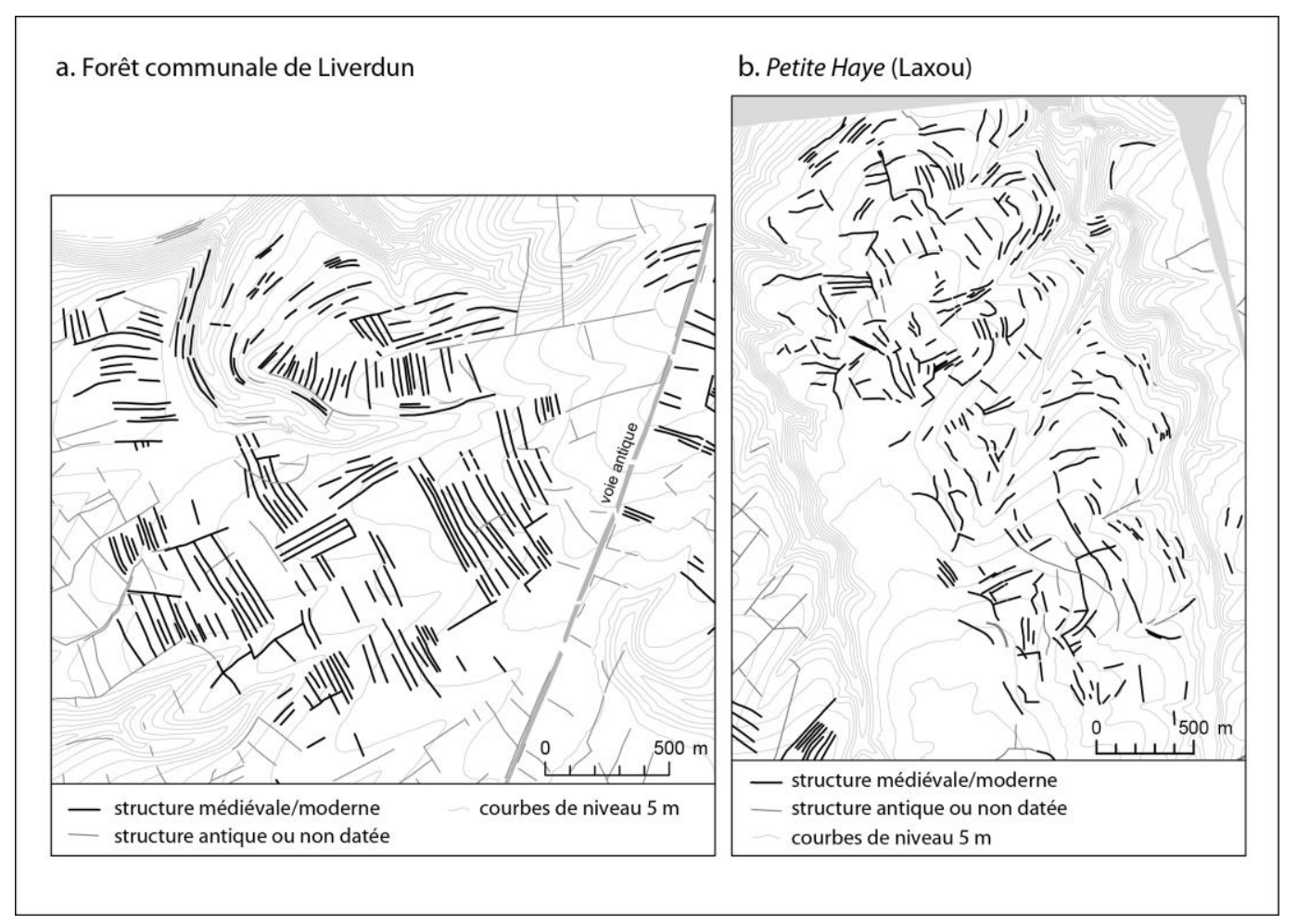

Figure 13. Parcellaire laniéré de Liverdun (a) et parcellaire "vermiforme " de la Petite Haye (b) dans la forêt de Haye (SIG/DAO MGL; données archéologiques : M. Georges-Leroy) 
Le second type se compose de bombements parallèles (champs bombés), conservés sur des longueurs de 80 à $250 \mathrm{~m}$, qui s'organisent en quartiers larges de 70 à $230 \mathrm{~m}$ (fig. 7a-b). Uniquement observés sur les marges de la forêt de Haye, ils sont conservés sur de petites surfaces. La plupart sont datés du XIX ${ }^{\mathrm{e}}$ et du début du $\mathrm{XX}^{\mathrm{e}}$ siècle d'après l'analyse des cartes d'état-major du XIX ${ }^{\mathrm{e}} \mathrm{s}$. et de plans et photographies aériennes du $X X^{\mathrm{e}} \mathrm{s}$.

\section{Des parcellaires «vermiformes »}

Toujours dans la forêt de Haye, des secteurs présentent des vestiges dont l'organisation est moins structurée, du moins dans les traces qui sont parvenues jusqu'à nous. Composés principalement de talus terreux, ces vestiges sont moins denses, ont des longueurs et des orientations variées et sont fréquemment courbes, voire sinueux. Ils peuvent s'organiser ponctuellement en lanières de 3 à 4 talus et sont implantés indifféremment dans le sens de la pente ou à son opposé. Ils se développent principalement dans le secteur de la Petite Haye, à Laxou, sur une croupe délimitée par deux vallons secs qui se rejoignent au nord, sur une surface de 430 ha (fig. 13b). Associés à ces talus, on note la présence fréquente des bourrelets de terre parallèles, interprétés comme des crêtes de labour (fig. 8). Pour les mêmes arguments que les parcellaires laniérés, ces parcellaires «vermiformes » sont datés de l'époque médiévale ou moderne (Georges-Leroy \& Zeller-Belville 2018). Quelques rares textes pourraient suggérer que l'on est en présence de traces liées à une mise en culture d'essarts en forêt durant une courte période au Moyen Âge, voire à des témoins de cultures par les habitants réfugiés en forêt lors des guerres du XVII ${ }^{\mathrm{e}}$ siècle.

\section{Des parcellaires cloisonnés viticoles}

Enfin, en périphérie du massif de Haye, sur des zones pentues, on peut observer une organisation en petites unités quadrangulaires délimitées par des murs en partie effondrés (fig. 14). La vigne y est attestée au début du XIX ${ }^{\mathrm{e}}$ s. d'après le cadastre napoléonien.

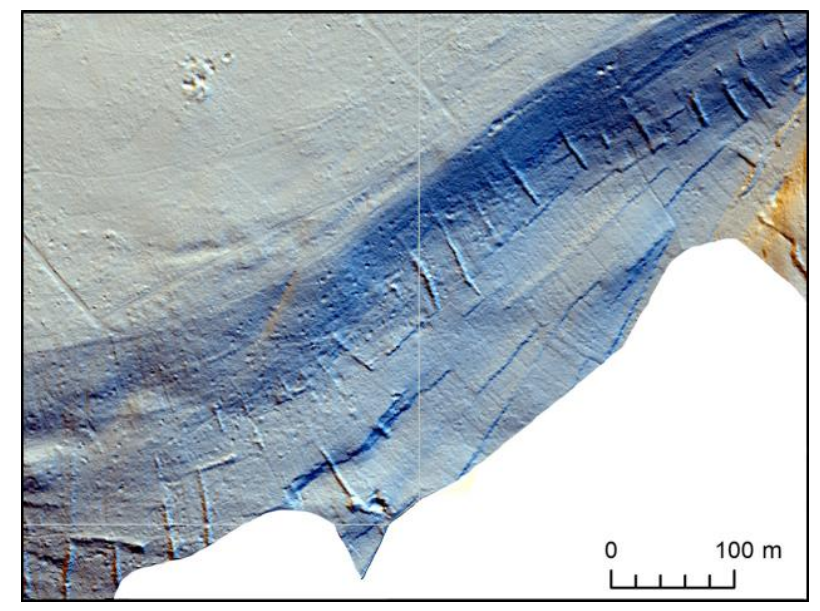

Figure 14. Murs de cloisonnements viticoles, visualisations par ombrage multidirectionnel (16 dir., az. $35^{\circ}-$ données LiDAR Haye 2007, DRAC Lorraine-INRA Nancy-ONF)

\section{Conclusion}

Ces modestes vestiges, parfois peu visibles au sol, mais bien mis en évidence par les acquisitions LiDAR, constituent une matière considérable pour l'étude des paysages anciens. Le LiDAR permet de les appréhender sur de vastes surfaces, ce qui facilite une approche morphologique des parcellaires, mais aussi une appréhension de l'occupation du sol à diverses périodes. Il fournit aussi des données sur la forme même des structures agraires et parcellaires, autorisant une étude de leur morphogenèse. Toutefois, pour progresser dans la compréhension de leur formation et des processus taphonomiques qui suivent leur abandon, la seule analyse des levés ne suffit pas. Il est en effet nécessaire pour cela de mener des interventions de terrain (fouilles, carottages, analyses pédologiques et 
micromorphologiques...). Seules des approches combinant analyse des levés LiDAR, opérations de terrain et études d'archives peuvent produire des éléments pour avancer dans l'élaboration de référentiels de comparaison, qui doivent porter sur différentes régions, sur différents types de sols ou sous divers couverts végétaux. Elles doivent aussi servir à progresser dans la datation de ces structures agraires et parcellaires, qui s'avère encore assez délicate.

\section{Bibliographie}

Blaising, J.-M. 2010. «La charrue, outil de modelage du paysage durant le dernier millénaire », in : M.-H. Benetière (éd), Le jardinier et ses outils. Paris, ministère de la Culture et de la Communication : 7-15 (4 Cahier du Conseil national des parcs et jardins).

Blanchemanche, P. 1990. Bâtisseurs de paysages. Terrassement, épierrements et petite hydraulique agricoles en Europe. $X V I I^{e}-X I X^{e}$ siècles. Paris, Éd. de la MSH, $329 \mathrm{p}$.

Callot, H.-J. 1980. La plaine d'Alsace. Modelé agraire et parcellaire. Nancy, université de Nancy II, 338 p.

Chouquer, G. 2006. «La question de l'émergence et de la mobilité de la planimétrie rurale à l'Âge du Fer », Les nouvelles de l'archéologie, 104-105: 72-79.

Chouquer, G. 2009. «Archéogéographie des trames planimétriques en Côte-d'Or », in: M. Provost (éd), Carte archéologique de la Gaule. La Côte-d’Or. 21/1 Alésia. Paris, Académie des inscriptions et belles lettres : $183-264$.

Chouquer, G. \& Favory, F. 2001. L’arpentage romain. Histoire des textes. Droit. Techniques. Paris, Éd. Errance, 491 p.

Comet, G. 1992. Le Paysan et son outil. Essai d'histoire technique des céréales (France, VIII'-XV siècle). Rome, École française de Rome, $711 \mathrm{p}$.

East Sussex County Council 2012. Feature Identification Charts. A toolkit for wooded landscapes. http://www.highweald.org/downloads/publications/land-management-guidance/woodland-1/33-feature-identificationtoolkit-final-feb-2012/file.html

Étienne, D., P. Ruffaldi, S. Goepp, F. Ritz, M. Georges-Leroy, B. Pollier \& É. Dambrine 2011. "The origin of closed depressions in Northeastern France: a new assessment”, Geomorphology, 126 : 121-131.

Étienne, D., P. Ruffaldi, J.-L. Dupouey, M. Georges-Leroy, F. Ritz \& É. Dambrine 2013. "Searching for ancient forests: A 2000 year history of land use in northeastern French forests deduced from the pollen compositions of closed depressions", The Holocene, 23(5) : 678-691.

Étienne, D., M. Destas, É. Lyautey, R. Marti, P. Ruffaldi, M. Georges-Leroy, É. Dambrine \& E. Topp 2015. “Two thousand-year reconstruction of livestock production intensity in France using sediment-archived fecal Bacteroidales and source-specific mitochondrial markers", The Holocene, 25(9) : 1384-1393.

Favory, F., 2011. «Les parcellaires antiques de l'Est de la Gaule », in : M. Reddé (éd), Aspects de la romanisation dans l'Est de la Gaule. Glux-en-Glenne, Bibracte : 385-416 (Bibracte 21).

Georges-Leroy, M., J. Bock, É. Dambrine \& J.-L. Dupouey 2012a. « Apport du LiDAR à la connaissance de l'histoire de l'occupation du sol en forêt de Haye », ArchéoSciences, 35-2011, p. 117-129.

Georges-Leroy, M., J. Bock, É. Dambrine, J.-L. Dupouey, A. Gebhardt \& J.-D. Laffite 2012b. «Les vestiges galloromains conservés dans le massif forestier de Haye (Meurthe-et-Moselle). Leur apport à l'étude de l'espace agraire », in: V. Carpentier \& C. Marcigny (éds), Des hommes aux champs. Pour une archéologie des espaces ruraux du Néolithique au Moyen Âge. Rennes, PUR : 157-180.

Georges-Leroy, M., N. Poirier, S. David, N. Meyer, F. Favory, É. Fovet \& P. Nouvel à paraître, «La dynamique des finages dans la longue durée », in : F. Bertoncello et al. (éds), ARCHAEDYN. Dynamique spatiale des territoires de la Préhistoire au Moyen Âge. Besançon, PUFC (Cahiers de la MSHE).

Georges-Leroy, M. \& S. Viller 2016. «Les apports du LiDAR à l'archéologie préventive : l'exemple de la LGV Est Paris-Strasbourg en Lorraine », Archéopages, Hors-série 4 : 82-91.

Georges-Leroy, M. \& C. Zeller-Belville 2018. «Des talus et du laser au service de l'histoire du paysage : les structures agraires médiévales et modernes révélées par le LiDAR dans le massif forestier de Haye (Meurthe-et-Moselle) », in : F. Journot (éd), Pour une archéologie indisciplinée. Réflexions croisées autour de Joëlle Burnouf. Drémil-Lafage, Éd. Monique Mergoil : 155-163 (Europe médiévale 12).

Gérard, F. 2012. «La structuration du village pour une économie agraire planifiée à la fin du IX ${ }^{\mathrm{e}}$ siècle en Lorraine. Les sites de Vitry-sur-Orne et de Demange-aux-Eaux », Archéopages, 34 : 38-47. 
Hall, D. 1982. Medieval fields. Aylesbury, Shire Publications, 56 p. (Shire archaeology).

Haudricourt, A.-G. \& M. Jean-Brunhes Delamare 2000. L'homme et la charrue à travers le monde. Tournai, La Renaissance du livre, 617 p.

Kokalj, Ž., K. Zakšek \& K. Oštir 2011. "Application of Sky-View Factor for the Visualisation of Historic Landscape Features in LiDAR-Derived Relief Models", Antiquity, 85 : 263-273.

Szabó, P. 2010. “Ancient woodlands boundaries in Europe”, Journal of Historical Geography, 36 : 205-214.

Zadora-Rio, É. 1991. «Les terroirs médiévaux dans le Nord et le Nord-Ouest de l'Europe », in : J. Guilaine (éd), Pour une archéologie agraire : à la croisée des sciences de l'homme et de la nature. Paris, A. Colin : 165-192. 